\title{
What will Al Ophthalmology v2.0 look like?
}

Louis R. Pasquale, MD, FARVO

Department of Ophthalmology, Icahn School of Medicine at Mount Sinai, New York, NY, USA

\section{Artificial intelligence: from 'gee whiz' to 'wow'}

It is fair to say that the field of artificial intelligence (AI) has moved from the 'gee whiz' phase to the 'wow' phase. In the 'gee whiz' phase, a computer could be programmed to play checkers as well as a human player. Jumping ahead to the 'wow' phase, convoluted neural networks (CNNs) facilitate the detection of clinically significant levels of glaucomatous optic neuropathy, retinopathy of prematurity, and diabetic retinopathy with precision that rivals and even exceeds that of skilled clinicians. CNNs are computer algorithms that are organized in ways that allow for layered processing of data with a hierarchical architecture that mimics the cerebral visual cortex. The FDA has approved two Al algorithms - IDx ${ }^{1}$ and EyeArt ${ }^{2}$ - for their ability to detect referable diabetic retinopathy as well as a reading center manned by experts using robust clinical standards. The new journal, Modeling and Artificial Intelligence in Ophthalmology, will chronicle Al Ophthalmology version 2.0. What will Al Ophthalmology v2.0 look like?

\section{Clinical care}

Programming languages with commands to perform the forward- and back-propagation steps that optimize the computer learning process are used to construct CNNs that power Al algorithms in medicine. CNNs are well suited to autonomously analyze ophthalmic images, opening the door to numerous opportunities to advance our field. In Al Ophthalmology version 2.0, we can expect Al to expand beyond diagnosing disease to identifying idealized therapeutic approaches to treat and forecast ophthalmic disease course. Furthermore, Al will be used in ophthalmology research to effectively discover metabolite and genetic profiles that differentiate patients with common complex eye diseases like age-related macular degeneration and glaucoma from controls. An interesting fusion between mathematical modeling and Al seems possible with the advent of optical coherence 
tomography angiography (OCTA). OCTA demonstrates unparalleled ability to resolve the various plexiform vascular beds in the retina and generate quantitative data on vessel density, vascular tortuosity, foveal avascular zone area, ${ }^{3}$ etc., enabling exciting possibilities of combined mathematical modeling and $\mathrm{Al}$ in understanding ophthalmic diseases with a retinal vascular component.

\section{Teaching}

As Al Ophthalmology version 2.0 evolves, there will be a need to create continuing medical education programs for trainees and practicing ophthalmologists to educate and update them about the field. It will also be worthwhile to reverse engineer the computer learning process to make practitioners better observers of clinical material and researchers better able to decipher the clues hidden in their complex datasets. We often hear the phrase, "a picture is worth a thousand words". The ability to be a keen observer of ophthalmic images is what makes ophthalmologists well suited to describe the retinal manifestations of systemic disease. Al has extended this power of observation to include chronological age prediction, ascertainment of sex, prediction of smoking status, and estimation of actual blood pressure levels. ${ }^{4}$ Learning how CNNs extend these powers of observation is certainly worthwhile. Interestingly, this process has already begun. Lessons learned thus far include that, among imaging modalities, OCT volume scans are superior to cross-sectional OCTs and fundus photos for age and sex prediction. ${ }^{5}$ Furthermore, fundus photos with both the optic nerve and macula are needed for sex prediction. Finally, the ability to make these predictions does depend on the existence of ophthalmic pathology and is also age dependent.

\section{Health equity}

Al offers a great opportunity to level the playing field in terms of providing access to care to all people in all corners of the world. The challenge in $\mathrm{Al}$ is that training algorithms typically requires large amounts of data and it is important that such datasets are representative of the diverse populations where they will be implemented. ${ }^{6}$ Furthermore, there is a need to create algorithms that work for images captured on a variety of platforms, including cell phones, which are readily available. Another trend that is already happening is the use of machineto-machine learning to train algorithms that predict OCT level retinal biomarker quantitative data from fundus photographs. ${ }^{7}$ The ability to convert a fundus camera into an instrument that could also extract OCT level data will be welcome in areas where OCT technology is not available or not affordable. Also, there is a need for cloud-based computing like auto machine learning to make Al available 
to interested physicians so that they can tailor algorithms that have utility in their own practice. ${ }^{8}$ Finally, since Al algorithms can function as well as reading centers, ${ }^{1}$ then there may be a reduced need for such centers in randomized clinical trials (RCTs) for conditions like rapidly progressive open-angle glaucoma, dry age-related macular degeneration, and anterior ischemic optic neuropathy. The reduced costs associated with supplanting reading centers with Al-based approaches could shunt resources devoted to the discovery of new neuroprotective agents for these conditions.

\section{Challenges}

There are several burning questions that extend beyond the 'wow factor' as we enter Al Ophthalmology v2.0:

1. Will there be a RCT showing that implementation of an Al algorithm results in preservation or improvement in vision?

2. How do we engage the next generation (and the current generation for that matter) of ophthalmologists to embrace Al in their practice given the inevitable penetration of this discipline into every aspect of medicine in general and ophthalmology in particular?

3. Will Al algorithms be able to 'multitask'? Currently, algorithms are designed to detect one outcome but will be totally agnostic to other coexistent but clinically important outcomes.

4. How will Al algorithms be maintained such that they robustly and faithfully perform their appointed tasks over time?

\section{Conclusion}

Ophthalmic researchers have been on the forefront of the Al revolution. The future for $\mathrm{Al}$ in ophthalmology is bright when one considers the merger of this field with disciplines like genomics, the microbiome, and integrated longitudinal medical records that incorporate systemic conditions. Al has the capability to improve care, make physicians better clinical observers and allow them to make more cost-effective medical decisions in the allocation of care. There is also the possibility that Al Ophthalmology v2.0 will work to assist our colleagues in the management of other neurodegenerative and neuropsychiatric diseases such as Alzheimer's disease and schizophrenia, as the retina is an accessible aspect of the central nervous system. 


\section{Declarations}

\section{Competing interests}

Dr. Pasquale is paid consultant to Twenty Twenty, Eyenovia, and Skye Biosciences.

\section{Funding}

Dr. Pasquale is supported by an unrestricted challenge grant from Research to Prevent Blindness (NYC, USA) and by grants from the NEI (R01 EY015473, R01 EY032559).

\section{References}

1. Abràmoff MD, Lavin PT, Birch M, Shah N, Folk JC. Pivotal trial of an autonomous Al-based diagnostic system for detection of diabetic retinopathy in primary care offices. NPJ Digit Med. 2018 Aug 28;1:39. https://doi.org/10.1038/s41746-018-0040-6

2. Bhaskaranand M, Ramachandra C, Bhat S, Cuadros J, Nittala MG, Sadda SR, Solanki K. The Value of Automated Diabetic Retinopathy Screening with the EyeArt System: A Study of More Than 100,000 Consecutive Encounters from People with Diabetes. Diabetes Technol Ther. 2019 Nov;21(11):635643. Epub 2019 Aug 7. https://doi.org/10.1089/dia.2019.0164

3. Yao X, Alam MN, Le D, Toslak D. Quantitative optical coherence tomography angiography: A review. Exp Biol Med (Maywood). 2020 Feb;245(4):301-312. Epub 2020 Jan 20. https://doi. org/10.1177/1535370219899893

4. Poplin R, Varadarajan AV, Blumer K, Liu Y, McConnell MV, Corrado GS, Peng L, Webster DR. Prediction of cardiovascular risk factors from retinal fundus photographs via deep learning. Nat Biomed Eng. 2018 Mar;2(3):158-164. Epub 2018 Feb 19. https://doi.org/10.1038/s41551-018-0195-0

5. Munk MR, Kurmann T, Márquez-Neila P, Zinkernagel MS, Wolf S, Sznitman R. Assessment of patient specific information in the wild on fundus photography and optical coherence tomography. Sci Rep. 2021 Apr 21;11(1):8621. https://doi.org/10.1038/s41598-021-86577-5

6. Campbell JP, Mathenge C, Cherwek H, Balaskas K, Pasquale LR, Keane PA, Chiang MF; American Academy of Ophthalmology Task Force on Artificial Intelligence. Artificial Intelligence to Reduce Ocular Health Disparities: Moving From Concept to Implementation. Transl Vis Sci Technol. 2021 Mar 1;10(3):19. https://doi.org/10.1167/tvst.10.3.19

7. Medeiros FA, Jammal AA, Thompson AC. From Machine to Machine: An OCT-Trained Deep Learning Algorithm for Objective Quantification of Glaucomatous Damage in Fundus Photographs. Ophthalmology. 2019 Apr;126(4):513-521. https://doi.org/10.1016/j.ophtha.2018.12.033

8. Faes L, Wagner SK, Fu DJ, Liu X, Korot E, Ledsam JR, Back T, Chopra R, Pontikos N, Kern C, Moraes G, Schmid MK, Sim D, Balaskas K, Bachmann LM, Denniston AK, Keane PA. Automated deep learning design for medical image classification by health-care professionals with no coding experience: a feasibility study. Lancet Digit Health. 2019 Sep;1(5):e232-e242. Epub 2019 Sep 5. https://doi. org/10.1016/S2589-7500(19)30108-6 